\title{
AKUPRESSUR, NEUROPHARMAKOLOGIE UND PSYCHISCHE GESUNDHEIT
}

\author{
Acupressure, Neuropharmacology and Mental Health
}

\section{Zusammenfassung}

Akupressur ist eine nicht pharmakologische Technik, die das Potenzial hat, verschiedene Krankheiten zu lindern. Diese Kurzübersicht beschäftigt sich mit Fakten wissenschaftlicher Akupressurstudien und fasst einige wichtige Aspekte zusammen. Wissenschaftliche Artikel aus den Datenbanken PubMed, Google Scholar und CNKI dienten als Forschungsdokumente. Darüber hinaus umfasste die Forschung zur Akupressur im Vergleich zu pharmakologischen Interventionen bei mentalen Erkrankungen eigenes Material, das bereits in wissenschaftlichen Artikeln veröffentlicht wurde. Insgesamt wurden 9 gelistete Publikationen identifiziert, von denen 8 als relevant angesehen wurden. Der Autor überprüfte den Inhalt auf Wertigkeit und diskutiert in diesem Artikel die Einschränkungen der nicht pharmakologischen Behandlungsmethode kritisch. Die verfuigbaren Beweise, dass Akupressur als zusätzliche nicht pharmakologische Methode zur Intervention bei mentalen Erkrankungen eingesetzt werden kann, sind spärlich. Es konnte keine evidenzbasierte Qualitätsstudie identifiziert werden, in der Akupressur mit pharmakologischen Behandlungen bei mentalen Erkrankungen direkt verglichen wird. Obwohl bei der Bewertung der alten traditionellen Akupressurmethode erhebliche Fortschritte erzielt wurden, werden in den kommenden Jahren weitere Forschungsarbeiten wie beispielsweise gut konzipierte randomisierte kontrollierte Studien erforderlich sein.

\section{Schlüsselwörter}

Akupressur, pharmakologische Interventionen, psychische Gesundheit, Traditionelle Chinesische Medizin, nicht pharmakologische Interventionen, Hirndruck, Schlaflosigkeit, Sedierung, COVID-19

\section{Summary}

Acupressure is a non-pharmacological technique that has the potential to relieve various diseases. This mini review deals with facts of scientific acupressure studies and summarizes some of important aspects. Scientific articles from the PubMed, Google Scholar and CNKI databases served as research documents. In addition, the research of acupressure versus pharmacologic interventions in mental diseases has included own material already published in scientific articles. A total of 9 listed papers were identified, 8 of which were considered relevant. The author checked the content for accuracy, and discussed limitations of the non-pharmacologic treatment modality critically. The evidence available that acupressure can be used as an additional non-pharmacological method of intervention in mental illness has been sparse. One could not identify any evidence-based high-level trial comparing acupressure with pharmacologic intervention in mental health care. Although considerable progress has been made in evaluating this ancient traditional method of acupressure, further research like large and well-designed randomized controlled trials will still be needed in the coming years.

\section{Keywords}

acupressure, pharmacological interventions, mental health, Traditional Chinese Medicine, non-pharmacological interventions, intracranial pressure, insomnia, sedation, COVID-19 


\section{Einleitung}

Die wissenschaftliche Literatur zu pharmakologischen Interventionen im Bereich der psychischen Gesundheit ist sehr umfangreich. Die Mechanismen und Wirkungen wurden dabei in verschiedenen Details nachgewiesen. Nicht pharmakologische Interventionen wie Akupressur oder Akupunktur wurden jedoch nicht in gleichem Ausmaß wissenschaftlich untersucht. Einige Studien zur Akupressur zwischen 2008 und 2021, die hauptsächlich biomedizinische Geräte verwenden und über die Auswirkungen von Akupressur und pharmakologischen Interventionen berichten, haben jedoch zu einem besseren Verständnis beigetragen und gezeigt, dass Akupressur erfolgreich, manchmal effizient und sicher bei psychischen Behandlungen eingesetzt werden kann. Seit den Anfängen der Akupressur bleiben jedoch immer noch viele Fragen zu den verschiedenen Methoden und Wirkungen dieser traditionellen nicht pharmakologischen Methode offen.

Dieser Artikel fasst in einem kurzen Überblick die wissenschaftlichen Erkenntnisse zum Thema Akupressur im Vergleich zu pharmakologischen Interventionen im Bereich mentaler Gesundheit zusammen. Aus den Fortschritten bei der Erforschung komplementärer Methoden könnten zusätzliche Therapieoptionen für die Patient`innen entstehen.

\section{Methoden}

\section{Suchstrategie}

Die Datenbanken von PubMed, Google Scholar und China National Knowledge Infrastructure (CNKI) wurden bis Anfang Februar 2021 durchsucht. Die bei der Suche verwendeten Booleschen Operatoren und Schlüsselwörter waren „Akupressur“, ,pharmakologische Intervention“, „,mentale Gesundheit“, „Akupressur UND mentale Gesundheit", ,pharmakologische Intervention UND mentale Gesundheit“, „Akupressur UND pharmakologische Intervention UND psychische Gesundheit“. Die Strategie und die Schlüsselwörter wurden entsprechend der jeweiligen Datenbank angepasst. Darüber hinaus wurden eigene Forschungsergebnisse zum Thema auszugsweise zusammengefasst.

\section{Datenbanksuche}

Die Suchanfrage in der PubMed-Datenbank ergab 5 Artikel, die vom Autor analysiert wurden (Tab. 1).

Der Schwerpunkt der Analyse lag auf der Interpretation nicht pharmakologischer (Akupressur) versus pharmakologische Interventionen in Artikeln zur mentalen Gesundheit. Nach dem Hinzufügen von 4 eigenen

\begin{tabular}{|l|c|c|c|}
\hline \multicolumn{4}{|l|}{ Tab. 1: Ergebnisse verschiedener Datenbanken } \\
\hline $\begin{array}{l}\text { Datenbank/Schlüssel- } \\
\text { worte }\end{array}$ & PubMed & $\begin{array}{l}\text { Google } \\
\text { Scholar }\end{array}$ & CNKI \\
\hline Acupressure & 1444 & 43300 & 2290 \\
\hline $\begin{array}{l}\text { Pharmacological } \\
\text { intervention }\end{array}$ & 773572 & 1560000 & 193581 \\
\hline Mental health & 393856 & 3210000 & 76034 \\
\hline $\begin{array}{l}\text { Acupressure AND mental } \\
\text { health }\end{array}$ & 53 & 15800 & 7832 \\
\hline $\begin{array}{l}\text { Pharmacological } \\
\text { intervention AND mental } \\
\text { health }\end{array}$ & 7939 & 613000 & 800 \\
\hline $\begin{array}{l}\text { Acupressure AND } \\
\text { pharmacological } \\
\text { intervention AND mental } \\
\text { health }\end{array}$ & 5 & 9310 & 80 \\
\hline
\end{tabular}

Forschungsbeiträgen wurden 8 Veröffentlichungen zur weiteren Überprüfung implementiert (Abb. 1).

\section{Geschichte der Akupressur Definition - Akupressur}

Akupressurstudien, die in den Datenbanken Science Citation Index (SCI) und PubMed angeführt sind, nehmen stetig zu. Insgesamt gibt es in PubMed (www.pubmed. gov) zum 2. Februar 2021 insgesamt 1444 Veröffentlichungen zu diesem Thema. Akupressur kann beispielsweise mit Druck des Fingers, des Ellbogens usw. mit Kreis-, Reiboder Knetbewegungen durchgeführt werden [1-8]. Bei der Akupressur wird manchmal schnell gedrückt, wobei der Daumen Druck ausübt (Abb. 2) und sich in einer sehr schnellen Bewegung löst [9].

\section{Frühe Akupressur in Asien}

Akupressur wird in China seit mehr als Tausenden von Jahren eingesetzt, um körperliche Beschwerden zu lindern und zu verhindern. Akupressur findet sich nicht nur in der traditionellen chinesischen Medizin (TCM), sondern auch in vielen anderen Kulturen derWelt. Erst in China wurde die Akupressur jedoch zu einem umfassenden Behandlungskonzept von körperlichen Symptomen und Schmerzen entwickelt, indem der Druck auf bestimmte Hautstellen ausgeübt wurde [10,11].

In einem der ältesten medizinischen Werken, dem Klassiker des Gelben Kaisers für Innere Medizin (,Huangdi Neijing“, ca. 100 v. Chr.), finden sich bereits Aufzeichnungen über Massagetechniken als Therapie für Schmerzen, 


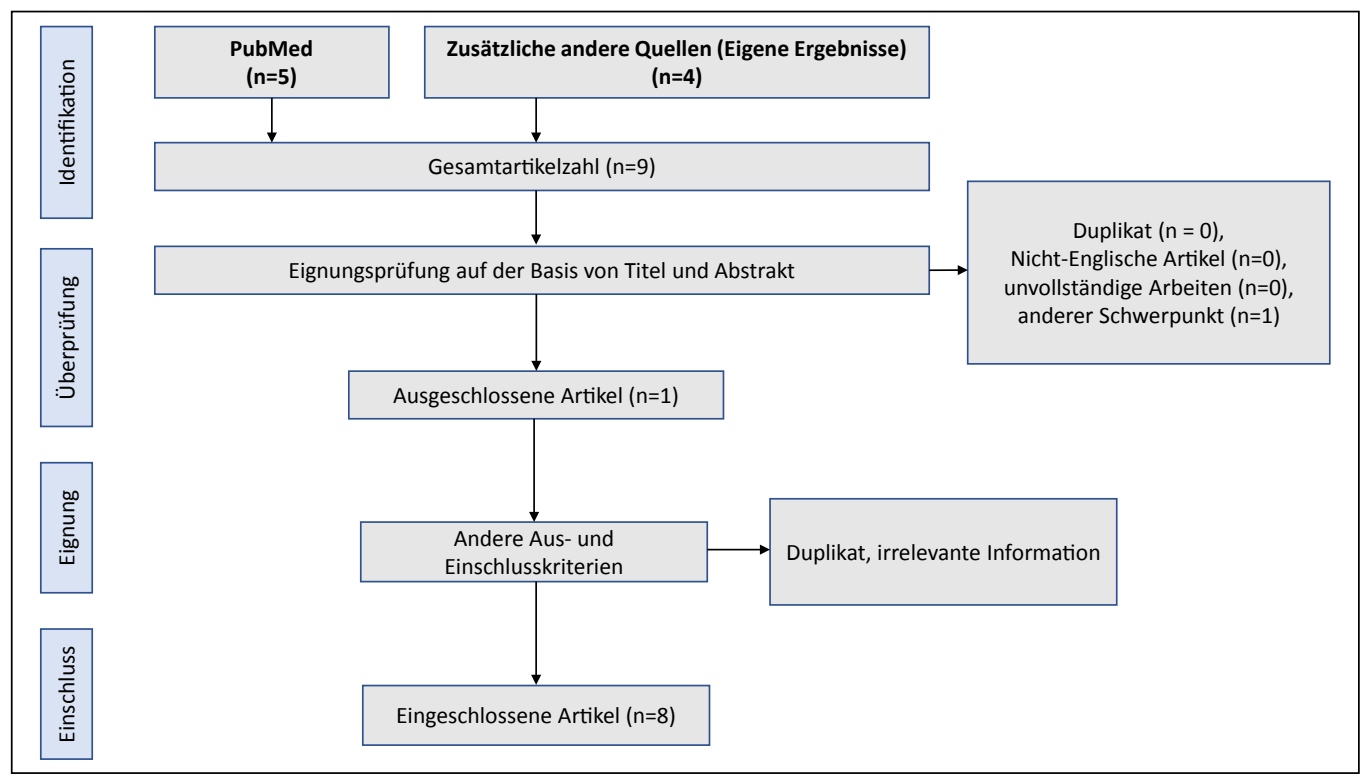

Abb. 1 Auswahl von Artikeln für diese Kurzübersicht

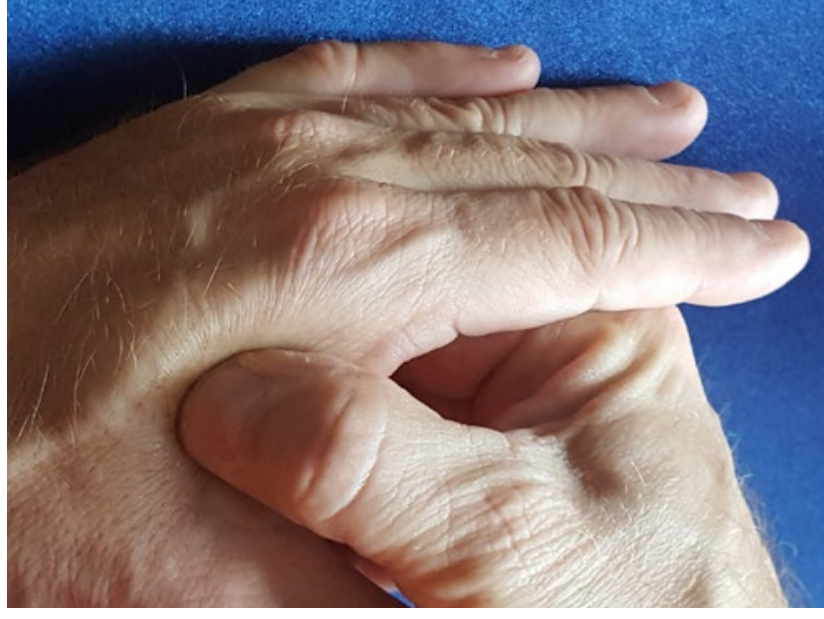

Abb. 2 Akupressur am Akupunkturpunkt Hegu (Di 4) mit dem Daumen

Energieblockaden und Muskelentspannung, die heute zum Teil noch Gültigkeit besitzen. Der chinesische Arzt Sun Simiao veröffentlichte die ersten genaueren Beschreibungen der Akupressur und ihrer Auswirkungen [12].

China ist das einzige Land der Welt, in dem westliche Medizin und TCM auf allen Ebenen des Gesundheitssystems nebeneinander praktiziert werden. Die TCM hat einen einzigartigen theoretischen und praktischen Ansatz zur Behandlung von Krankheiten, der sich über Jahrtausende entwickelt hat [13].

\section{Frühe Quellen der Akupressur in der westlichen Welt}

Akupressur bedeutet simplifiziert, Druck auf die Akupunkturpunkte auszuüben. Im Westen sind Akupressur und Akupunktur sehr oft auch mit Analgesie verbunden, wobei ihre Effektivität durch plausible biologische Mechanismen bez. Wirkung, wie etwa Freisetzung von Endorphin, zustande kommt. Die Methode wurde auch für dieVerwendung in der Anästhesie herangezogen, wird aber derzeit nur selten gerade dafür eingesetzt [13].

Die Ohrakupressur ist eine der ältesten Spezialformen der Akupressur und stellt ein Konzept der Somatopie dar, welches die differenzierte Abbildung des gesamten Körpers auf eine Körperregion, in diesem Fall die Ohrmuschel, beschreibt. Bereits das vorhin schon erwähnte Buch des Gelben Kaisers über Innere Medizin („Huangdi Neijing“) wies auf die Zusammenhänge zwischen Ohrmuschel und inneren Organen hin. Neben der chinesischen Medizin berichteten Hippokrates und die alte Medizin Ägyptens über ähnliche Befunde [14].

Die Ohrakupunktur wurde 1950 durch den französischen Arzt Paul Nogier zu einem umfassenden Aurikulomedizinsystem. 1959 wurde Nogiers Wissen über Ohrakupunktur und ihre therapeutischen und analgetischen Wirkungen auch in China bekannt und als Ergebnis in den traditionellen chinesischen Akupunkturunterricht als Mikrosystem integriert $[15,16]$.

\section{Frühere und aktuelle Akupressurforschung}

Die philosophische Tradition der chinesischen Medizin enthält ein starkes präventives Element, das eng mit dem Konzept einer ausgewogenen Beziehung zwischen Mensch und Natur und zwischen Körper und Geist verbunden ist. Immer wird betont, dass ein gesunder Geist in einem gesunden Körper für das Wohlbefinden wesentlich ist. In der TCM gibt es eine Reihe von Ansätzen zur Gesundheitserhaltung. Der präventive Wert der Akupressur etwa am 
Punkt Zusanli (Ma 36) wird bereits 1982 in amerikanischen Fachzeitschriften diskutiert [14]. Akupressur wird auch als hilfreich angesehen, um die allgemeine (geistige) Gesundheit älterer Menschen zu verbessern und zu erhalten [14].

1994 berichtete Ortego, dass die orientalische Gesundheitsphilosophie eine Wissensbasis für Krankenschwestern bietet, die Akupressur als alternative Wahl für die Gesundheitsversorgung anbieten. Akupressur liefert einen KörperGeist-Ansatz zur Schmerzbehandlung und eine einzigartige Behandlungsmethode in der psychosozialen Betreuung [17]. In den folgenden Jahren zwischen 2006 und 2021 wurden weltweit die ersten klinisch-wissenschaftlichen Veröffentlichungen zu Akupressur und psychischer Gesundheit (mehr als 50 wissenschaftliche, zumeist evidenzbasierte Artikel, s. PubMed Tab. 1) veröffentlicht.

Insgesamt 1444 Studien (Stand Anfang Februar 2021) finden sich in PubMed, wenn man nach dem Thema ,Akupressur" sucht. Die häufigsten Studien wurden zu folgenden Bereichen verfasst: postoperative Übelkeit und Erbrechen (PONV), Dysmenorrhoe, durch Chemotherapie verursachte Übelkeit und Erbrechen, Schmerzen und Schlaflosigkeit. Die am häufigsten verwendeten Akupressurpunkte sind dabei Neiguan (KS 6) und „Milz 6“ (Mi 6). Die Studienumfrage zeigt, dass die meisten Veröffentlichungen in China und Regionen Chinas wie Taiwan, den USA und Korea durchgeführt wurden. Der Schwerpunkt lag auf der Entwicklung verschiedener Akupressurindikationen, Tierversuche, Fallberichte, Grundlagenforschung und klinischer Studien. Die meisten dieser Untersuchungen wurden mit Einzelpunktstimulationstechniken durchgeführt.

Hier sollte erwähnt werden, dass die Praxis der Akupressur in China während der COVID-19-Pandemie auch in Wuhan und anderen Städten in China sehr häufig angewendet wurde [18-20]. Akupunkturpunktanwendung, Pressen von sogenannten Samenkörnern auf Ohrakupunkturpunkten, Massage usw. waren in der Praxis während der Pandemie weit verbreitet. Die allgemein empfohlenen Akupunkturpunkte für die Akupunkturpunktanwendung (Akupressur) bei COVID-19 sind Zusanli (Ma 36), Guanyuan (KG 4), Dazhui (LG 14), Fengmen (Bl 12) und Feishu (Bl 13), und andere Akupunkturpunkte wie Zhongwan (KG 12) und Shenque (KG 8). Die Akupressurtherapie verbessert die Symptome wie Appetitlosigkeit, Husten, Schlaflosigkeit oder Kopfschmerzen der COVID19-Patient`innen wirksam [18-20].

\section{Diskussion}

Die Akupressur wird zum Beispiel bei Schmerzen im Allgemeinen, Neuropathie, Rückenschmerzen, schmerzhaften
Wehen, Migräne, Depressionen, Angstzuständen und Schlaflosigkeit angewendet. Darüber hinaus gibt es andere psychische Erkrankungen wie Schizophrenie und Demenz, Schlaganfall und andere Beschwerden als wesentliche Einsatzgebiete.

Hier sollte erwähnt werden, dass Akupressur und Shiatsu in der Literatur häufig synonym verwendet werden. Es gibt jedoch einige wichtige technische Unterschiede zwischen den beiden, einschließlich der Art des ausgeübten Drucks, der Art und Weise, wie der Daumen positioniert wird, und wie beispielsweise das Körpergewicht eingesetzt wird. Zum gegenwärtigen Zeitpunkt ist aus Sicht wissenschaftlicherVeröffentlichungen der Einfluss dieser Unterschiede in der Anwendung auf die Wirksamkeit dieser Techniken nicht bekannt. Es sollte jedoch üblich sein, dass

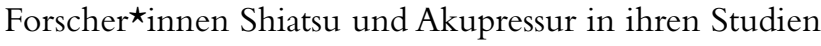
trennen und unterschiedliche Designs bzw. Gruppen auswählen, um korrekte Untersuchungen durchzuführen [9]. Daher sollten Forscher ${ }^{\star}$ innen, Praktiker ${ }^{\star}$ innen und andere Interessengruppen Studien, in denen die Wirksamkeit der Akupressur als Beweis für die Wirksamkeit von Shiatsu und umgekehrt beschrieben wird, nicht gegenseitig verwenden [9].

Pandian u. Mitarb. berichteten 2011 über alternative bzw. komplementäre Therapien zur Schlaganfallbehand-

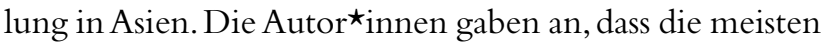
ergänzenden Therapien in der Rehabilitation von unbewiesenem Nutzen sind [21]. Wang u. Mitarb. untersuchten 2008 Akupunktur und auch Akupressur in der Depressionstherapie [22]. Eine Metaanalyse von 8 randomisierten kontrollierten Studien (RCTs) wurde interpretiert. RCTs, in denen 477 Proband $\star_{\text {innen verglichen wurden, wurden }}$ in die Metaanalyse einbezogen. Die Ergebnisse bestätigten, dass Akupressur und Akupunktur den Schweregrad der Depression signifikant reduzieren können, was durch verringerte Werte der Hamilton-Bewertungsskala für Depressionen (HAMD) oder im Beck Depression Inventory (BDI) angezeigt wurde. Innerhalb eines RCT haben Fogarty u. Mitarb. 2013 Patient ${ }^{\star}$ innen mit Anorexia nervosa, die Akupressur erhielten, untersucht [23]. Die Evidenz für bestehende psychologische und pharmakologische Interventionen zur Behandlung von Anorexia nervosa war nicht hoch, und dieVerwendung von Zusatztherapien, die die Wirksamkeit bestehender Behandlungen verbessern und damit zu verbesserten Ergebnissen für die psychische

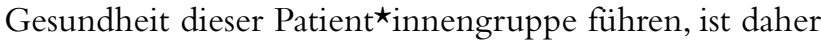
äußerst wünschenswert [23]. Die Studienteilnehmer^innen betrachteten die therapeutische Beziehung und Empathie als wichtige Eigenschaften der Akupressurintervention als 

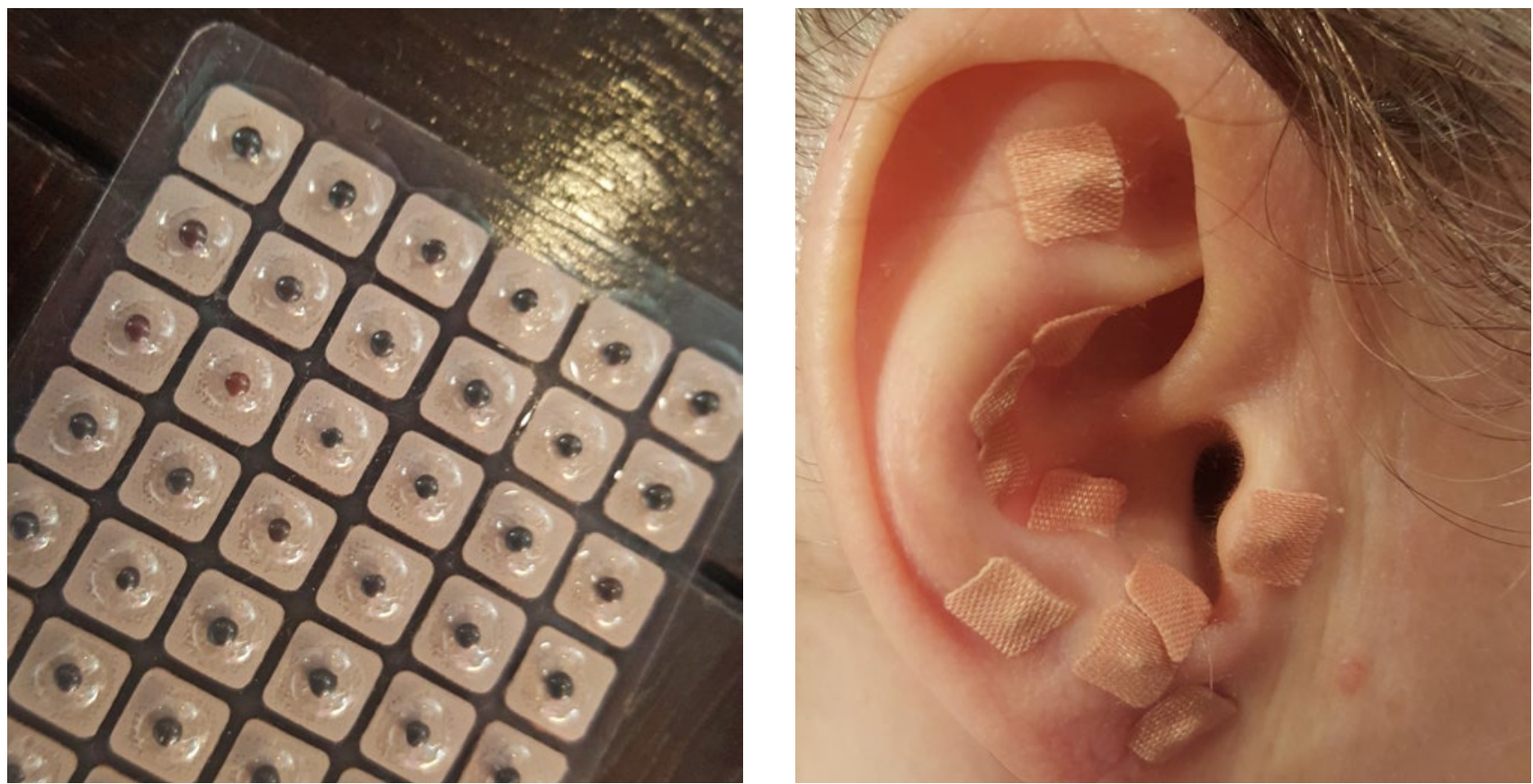

Abb. 3 Akupressur-Samenkörner (a) und praktische Anwendung am Ohr (b) bei Schlaflosigkeit. Selbstinduzierte Akupressur kann mit dieser Methode einfach durchgeführt werden.

Zusatztherapie zur Behandlung von Anorexia nervosa [23]. Im Jahr 2010 berichten Ebrahimi u. Mitarb. über ein optimales Management von Übelkeit und Erbrechen während der Schwangerschaft mit nicht pharmakologischen Ansätzen, derVerwendung von Ingwer, Akupressur,Vitamin B6 und Ernährungsumstellungen. Eine positive Wirkung dieser nicht invasiven, kostengünstigen und sicheren Methoden, einschließlich der Akupressur, wurde nachgewiesen [24]. Natürlich sind auch dazu pharmakologische Behandlungen mit unterschiedlicher Wirksamkeit verfügbar [24].

Um einen kurzen Einblick in ausgewählte Bereiche der wissenschaftlichen Erforschung der Akupressur im eigenen Forschungsbereich des Autors zu geben [25-27], wurden im Folgenden Schwerpunkte herangezogen, die mehrheitlich in den Bereich der Neurowissenschaften fallen. Obwohl es nur eine sehr begrenzte Datenmenge gibt, war ein Vergleich der nicht pharmakologischen Akupressur und der pharmakologischen Wirkungen in beschreibender Form möglich, jedoch nicht quantitativ in den einzelnen Grundlagenforschungsanwendungen.

Die Hauptbereiche sind: mögliche Erhöhungen des

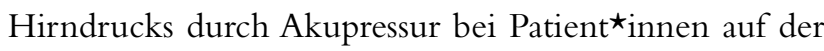
Intensivstation, der Einsatz von Akupressur bei Schlaflosigkeit und Sedierungseffekte durch Akupressur im Vergleich zu Anästhetika sowie Akupressur zur Linderung von Schmerzen und Müdigkeit bei COVID-19-Impfreaktionen und -nebenwirkungen.

\section{Hirndruck und manuelle Akupressur}

Litscher u. Mitarb. berichteten bereits 2005, dass der Druck auf die Akupunkturpunkte Xiaguan (Ma 7) und Erheliao (3E 22) bei Patient $\star$ innen mit erhöhtem intrakraniellen Druck (ICP) zu einem signifikanten Anstieg des reversiblen Hirndrucks führen kann [28]. Im Rahmen dieser Studie wurde erstmals untersucht, ob Veränderungen der mit dem ICP vergesellschafteten zerebralen Parameter auch bei gesunden Testpersonen festgestellt werden können, wenn die genannten Akupunkturpunkte durch Druck stimuliert werden. Insgesamt wurden 34 Proband $\star^{\star}$ innen und ein 15-jähriger Intensivpatient nach einem schweren Schädel-Hirn-Trauma untersucht. Die wichtigsten Bewertungsparameter waren die mittlere Blutflussgeschwindigkeit in der mittleren Hirnarterie (links und rechts) und der Pulsatilitätsindex. Zusätzlich wurden Parameter der Nahinfrarotspektroskopie und des Blutdrucks aufgezeichnet. Akupressur führte in einigen Fällen zu signifikanten Änderungen der Hauptzielparameter. Festgestellt wurde, dass Akupressur reproduzierbare funktionelle Veränderungen im Gehirn verursachen kann. Die Auswirkungen sollten insbesondere bei Patient ${ }^{\star}$ innen mit kraniozerebralem Trauma nicht vernachlässigt werden [28].

\section{Schlafstörungen und Akupressur}

Reza u. Mitarb. [29] untersuchten die Wirksamkeit der Akupressur auf die Schlafqualität mittels RCTs. Der Fragebogen „Pittsburgh Sleep Quality Index“ wurde als Screening-Tool 
bei 90 Senior ${ }^{\star}$ innen mit mittelschweren bis sehr schweren Schlafstörungen verwendet. Die Personen wurden randomisiert und einer Akupressurgruppe, einer Scheinakupressurgruppe und einer Kontrollgruppe zugeordnet. Die Ergebnisse zeigten signifikante Unterschiede zwischen der Akupressurgruppe und der Kontrollgruppe in Bezug auf Schlafqualität, Dauer und Effizienz. Es wurden keine signifikanten Unterschiede zwischen Scheinakupressurgruppe und Kontrollgruppe gefunden. In der Akupressurgruppe wurde eine deutliche Abnahme des nächtlichen Erwachens festgestellt. Die Ergebnisse dieser Studien legen nahe, dass Akupressur die Schlafqualität verbessert und eine nicht pharmakologische Zusatzmethode zu pharmakologischen Interventionen sein könnte. Eine taiwanesische Studie beobachtete, dass Akupressur am Punkt Shenmen (He 7) die Schlafqualität bei älteren Menschen bis zu 2 Wochen nach der Akupressurbehandlung verbesserte [30]. Cerrone u. Mitarb. [31] veröffentlichten eine weitere Studie, die die Wirksamkeit des untersuchten Akupunkturpunkts Shenmen (He 7) zeigt. Insgesamt nahmen 25 Patient ${ }^{\star}$ innen mit Schlafstörungen teil, von denen 14 Krebs hatten. Mindestens 2 Wochen lang wurden die Testpersonen am He 7-Punkt gegen Schlaflosigkeit behandelt. Bei 15 von 25 Testpersonen wurde eine Verbesserung der Schlafqualität festgestellt. Abb. 3 zeigt Akupressur-Samenkörner und Punkte am Ohr, die bei Schlaflosigkeit verwendet werden können.

\section{Sedierung durch Akupressur}

Ein Ziel einer anderen Studie von Litscher im Jahr 2004 war es, die Auswirkungen der Akupressurstimulation auf den elektroenzephalografischen (EEG) Bispektralindex (BIS), die spektrale Eckfrequenz (SEF) und einen geschätzten Sedierungswert zu untersuchen [3]. 25 gesunde Freiwillige wurden im Wachzustand untersucht. Der AkupunkturpunktYintang (EX-HN 3) und ein Placebo-Kontrollpunkt wurden stimuliert. Die Studie wurde als RCTund teilweise verblindete Crossover-Studie durchgeführt. Sowohl die BIS- als auch die SEF-Werte nahmen während der Akupressur am PunktYintang signifikant ab. Die nicht pharmakologische Intervention reduzierte auch den auf einer Skala eingetragenen Sedierungswert signifikant. Unsere Studie beleuchtet die elektroenzephalografischen Ähnlichkeiten von akupressurinduzierter Sedierung und gerätetechnischer „State of the Art"-Narkose, wie sie von BIS und SEF bewertet wurden [3]. Die Ergebnisse wurden von anderen Autoren bestätigt [32, 33]. Kim u. Mitarb. führten eine Studie an Zwergschnauzerhunden durch. Die Akupunkturpunkte Baihui (LG 20) und Yintang (EX-HN 3) wurden für die Dauer von 20 min stimuliert. Das Sedie- rungsniveau wurde vor, während und nach der Akupunktur anhand der SEF-Werte und des Ramsay-Sedierungsscores (RSS) bewertet. Die SEF-Werte waren während der Akupunktur am Baihui- oderYintang-Punkt signifikant verringert und kehrten nach der Akupunkturbehandlung zu den Grundwerten zurück. Der RSS zeigte auch ein akzeptables Sedierungsniveau. Die Anwendung der nicht pharmakologischen Intervention am Baihui- oderYintangPunkt in der vorliegenden Studie könnte eine wertvolle Methode sein, um die Sedierung bei Hunden zu induzieren [32]. Fassoulaki u. Mitarb. [33] führten eine weitere Studie zum Thema Akupressur zum Punkt Yintang durch. Die Autor ${ }^{\star}$ innen untersuchten die Werte für BIS, Melatonin, Beta-Endorphin und verbalen Stress vor, nach $10 \mathrm{Mi}-$ nuten Akupressur am Yintang-Punkt, an einem Kontrollpunkt, nach keiner Akupressur und 1 Stunde nach Abschluss der Intervention bei 12 Freiwilligen. Die BIS- und verbalen Stress-Score-Werte waren nach Akupressur verringert, aber Melatonin und Beta-Endorphin änderten sich nicht. Akupressur am Yintang-Punkt hatte somit keinen Einfluss auf den Melatonin- und Beta-Endorphinspiegel.

\section{Akupressur und Laserakupunktur zur Linderung von Schmerzen und Müdigkeit bei COVID- 19-Impfreaktionen und -nebenwirkungen}

Im Jahr 2020 gingen alleine in Deutschland 305641 Lebensjahre durch COVID-19 verloren. Personen, die an COVID-19 gestorben sind, haben durchschnittlich 9,6 Lebensjahre verloren; diejenigen, die zum Zeitpunkt ihres Todes unter 70 Jahre alt waren, verloren durchschnittlich 25,2 Lebensjahre. Männer verloren mehr Lebensjahre als Frauen (11,0 vs. 8, 1 Jahre). Zu diesem Schluss kommt eine aktuelle Studie aus Deutschland [34].

Aus der Sicht des Autors dieses Artikels ist es richtig, sich impfen zu lassen. Nicht wenige Menschen haben allerdings auch Sorge vor starken Impfreaktionen und/oder -nebenwirkungen. Die Befürchtung liegt oft vor, sie muss aber nicht eintreffen. Konventionelle Fieber- und Schmerzmittel können hierfür eine Hilfe sein.Viele Patient ${ }^{\star}$ innen möchten aber möglichst keine konventionellen Pharmaka auf chemischer Basis nehmen.

Derzeit (März 2021) wird gerade in umfassenden Studien untersucht, inwieweit etwa Ohrakupressur zur Linderung von Schmerzen (einschließlich Schmerzen an der Injektionsstelle, Kopfschmerzen, andere Muskel- und Gelenkschmerzen), Müdigkeit und eventuell gastrointestinalen Nebenwirkungen (einschließlich Übelkeit und/oder Erbrechen) nach Injektion neuartiger COVID-19-Impfstoffe beitragen kann [35]. 
Die Studie ist als multizentrische, dreiarmige, einfachblinde, prospektive, randomisierte Parallelgruppenstudie (Verhältnis 1:1:1) mit mehreren Gruppen konzipiert. Mehr als 360 Teilnehmer ${ }^{\star}$ innen im Alter von 18-59 Jahren werden eingeschlossen. Die Personen der Akupressurgruppe erhalten 5 Tage lang bilaterale Stimulation an 5 Ohrpunkten, 3- bis 4-mal (jeweils ca. $1 \mathrm{~min}$ ) Selbstakupressur pro Tag nach der Injektion (insgesamt 10 Tage). Zusätzlich erfolgen Beurteilungen einer Sham-Akupressurgruppe und einer weiteren Kontrollgruppe. Die klinische Studie wurde am 8. Februar 2021 registriert [35].

Insgesamt gibt es eine große Zahl von Studien zur Akupressur zu einerVielzahl von Themen. DieVeröffentlichungen legen nahe, dass Akupressur bei bestimmten Indikationen wie Chemotherapie-induzierte Nausea und Emesis (CINV), PONV, Dysmenorrhoe und Schlafstörungen hochwirksam sein kann. Als nicht invasive und zusätzliche ergänzende Therapie zur Pharmakologie bietet Akupressur aufgrund ihrer einfachen Anwendung und Kosteneffizienz ein gewisses Potenzial. Letztendlich gibt es wissenschaftliche Beweise für eine gewisse Wirksamkeit der Akupressur, jedoch sind aus wissenschaftlicher Sicht noch viele weitere Untersuchungen erforderlich.

Eine Schwachstelle vieler Studien ist die Studiendauer und Häufigkeit der Akupressurbehandlung, die in vielen Studien nicht angegeben wird. Daher sind aus vielen Studien keine objektiv messbaren Ergebnisse verfügbar. Effizienz und Wirkung wurden in vielen Untersuchungen subjektiv bewertet oder waren abhängig von der Interpretation, was das Risiko einer Fehlinterpretation birgt. Der-

\section{Literatur}

[1] Agarwal A, Ranjan R, Dhiraaj S et al.: Acupressure for prevention of pre-operative anxiety: a prospective, randomised, placebo-controlled study. Anaesthesia 2005; 60: 978-981.

[2] Wu HS, Wu C, Lin JG et al.: Effectiveness of acupressure in improving dyspnoea in chronic obstructive pulmonary disease. J Adv Nurs 2004; 45: 252-259.

[3] Litscher G: Effects of acupressure, manual acupuncture and laserneedle acupuncture on EEG bispectral index and spectral edge frequency in healthy volunteers. Eur J Anaesthesiol 2004; 21: 13-19.

[4] Lang T, Hager H, Funovits V et al.: Prehospital analgesia with acupressure at the Baihui and Hegu points in patients with radial fractures: a prospective, randomized, double-blind trial. Am J Emerg Med 2007; 25: 887-893. zeit sind keine Doppelblindstudien möglich. Manchmal fehlte eine Kontrollgruppe oder Placebogruppe und/oder eine kleine Anzahl von Proband $\star_{i n n e n}$ wurden eingeschlossen. Sehr oft wurde die Interpretation der Ergebnisse verfälscht, wenn während der Studie Medikamente verwendet wurden. Es fehlten auch Vergleiche zwischen kurzfristigen und langfristigen Auswirkungen.

Obwohl Akupressur im Vergleich zu anderen evidenzbasierten komplementärmedizinischen Methoden eine relativ lange Tradition hat, hat dieVerwendung von Druck als therapeutische Methode noch nicht so weit in der Vergangenheit begonnen.

Heute begleiten innovative Methoden nach dem neuesten Stand der Technik zukünftige Forschungsstrategien im Bereich der Akupressur. Dies schließt die Entwicklung moderner Stimulationsmethoden oder die Robotik ein.

\section{Schlussfolgerung}

Die alte traditionelle Methode der Akupressur hat bereits eine umfassende Geschichte hervorgebracht. Erkenntnisse aus der medizinischen Grundlagenforschung und der klinischen Forschung haben dazu beigetragen, die Auswirkungen der Akupressur auf biomedizinischer und klinischer Basis zu erklären, die für eine westliche medizinische Anerkennung akzeptabel sind. Der Autor ist der Meinung, dass Akupressur mit konventioneller Medizin unter Verwendung der Pharmakologie kombiniert werden könnte.

\section{Interessenskonflikt}

Der Autor erklärt, dass kein Interessenskonflikt besteht.
[5] Cho YC, Tsay SL:The effect of acupressure with massage on fatigue and depression in patients with end-stage renal disease. J Nurs Res 2004; 12: 51-59.

[6] Dullenkopf A, Schmitz A, Lamesic G et al.: The influence of acupressure on the monitoring of acoustic evoked potentials in unsedated adult volunteers. Anesth Analg 2004; 99:1147-1151.

[7] Chen LL, Hsu SF, Wang MH et al.: Use of acupressure to improve gastrointestinal motility in women after trans-abdominal hysterectomy. Am J Chin Med 2003; 31: 781-790.

[8] Jin KK, Chen L, Pan JY et al.: Acupressure therapy inhibits the development of diabetic complications in Chinese patients with type 2 diabetes. J Altern Complement Med 2009; 15: 1027-1032.

[9] Cabo F, Baskwill A, Aguaristi I et al.: Shiatsu and Acupressure: Two different and distinct techniques. Int $\mathrm{J}$ Ther Massage Bodywork 2018; 11: 4-10. 
[10] MichenthalerY: Scientific basics of acupuncture. Thesis, Medical University of Graz, 2012. Deutsch

[11] Zhou D, LiuYY, Li GL et al.: Summarization and analysis on acupuncture-related articles embodied in Medline data-base in 2006. Zhongguo Zhen Jiu 2008; 28: 151-155. Chinesisch

[12] Song ZM: Historical origin of Jiu Tangshu biography of Sun Simiao. Zhonghua Yi Shi Za Zhi 2012; 42: 264-271. Chinesisch

[13] Hesketh BT and Zhu WX: Health in China.Traditional Chinese medicine: one country, two systems. BMJ 1997; 315: 115-117.

[14] Zhuo DH: Preventive geriatrics: an overview from Traditional Chinese Medicine. Am J Chin Med 1982; 10: 32-39.

[15] Nogier P: Le réflexe auriculocardiaque (RAC). Lyon Méditeranée Medical: Paris, France, 1972; 8: 1709-1738.

[16] Nogier R: How did Paul Nogier establish the map of the ear? Med Acupunct 2014; 26: 76-83.

[17] Ortego NE: Acupressure: an alternative approach to mental health counseling through bodymind awareness. Nurse Pract Forum 1994; 5: 72-76.

[18] Li M,Yang X, Li K et al.: Traditional Chinese Medicine for novel coronavirus pneumonia treatment: main force or supplement? TMR Integrative Medicine 2020; 5: 62-64.

[19] Liang FX, Litscher G: COVID-19 (coronavirus disease-19): traditional Chinese medicine including acupuncture for alleviation - a report from Wuhan, Hubei province in China. OBM Integrative and Complementary Medicine 2020; 5: 4.

[20] Litscher G: Effectiveness of integrative medicine in COVID-19? Med Acupunct 2020; 32: 131-132.

[21] Pandian JD, Liu M, Misbach J et al.: Alternative therapies for stroke treatment in Asia. Int J Stroke 2011; 6: 541-543.

[22] Wang H, Qi H, Wang BS et al.: Is acupuncture beneficial in depression: a meta-analysis of 8 randomized controlled trials? J Affect Disord 2008; 111: 125-134.

[23] Fogarty S, Smith CA, Touyz S et al.: Patients with anorexia nervosa receiving acupuncture or acupressure; their view of the therapeutic encounter. Complement Ther Med 2013;21: 675-681.

[24] Ebrahimi N, Maltepe C, Einarson A: Optimal management of nausea and vomiting of pregnancy. Int J Womens Health 2010; 2: 241-248.

[25] Wang L, Cheng W, Sun Z et al.: Ear acupressure, heart rate, and heart rate variability in patients with insomnia. Evid Based Complement Alternat Med 2013; 2013: 763631.
[26] Litscher G, Ofner M, He W et al.:Acupressure at the meridian acupoint Xiyangguan (GB33) influences near-infrared spectroscopic parameters (regional oxygen saturation) in deeper tissue of the knee in healthy volunteers. Evid Based Complement Alternat Med 2013; 2013: 370341.

[27] Gao XY, Wang L, Gaischek I et al.: Brain-modulated effects of auricular acupressure on the regulation of autonomic function in healthy volunteers. Evid Based Complement Alternat Med 2012; 2012: 714391.

[28] Litscher G, Wang L, Schwarz G et al.: Increases of intracranial pressure and changes of blood flow velocity due to acupressure, needle and laser needle acupuncture? Forsch Komplementärmed Klass Naturheilkd 2005; 12:190-195. Deutsch

[29] Reza H, Kian N, Pouresmail Z et al.: The effect of acupressure on quality of sleep in Iranian elderly nursing home residents. Complement Ther Clin Pract 2010; 16: 81-85.

[30] Sun J, Sung M, Huang $M$ et al.: Effectiveness of acupressure for residents of long-term care facilities with insomnia: a randomized controlled trial. Int J Nurs Stud 2010; 47: 798-805.

[31] Cerrone R, Giani L, Galbiati B et al.: Efficacy of HT 7 point acupressure stimulation in 56 the treatment of insomnia in cancer patients and in patients suffering from disorders other than cancer. Minerva Med 2008; 99: 535-537.

[32] Kim MS, Nam TC: Electroencephalography (EEG) spectral edge frequency for assessing the sedative effect of acupuncture in dogs. J Vet Med Sci 2006; 68: 409-411.

[33] Fassoulaki A, Paraskeva A, Kostopanagiotou G et al.:Acupressure on the extra 1 acupoint: the effect on bispectral index, serum melatonin, plasma beta-endorphin, and stress. Anesth Analg 2007; 104: 312-317.

[34] Rommel A, Lippe EV, Plass D et al.:The COVID-19 disease burden in Germany in 2020 - years of life lost to death and disease over the course of the pandemic. Dtsch Arztebl Int 2021; 118: 145-151.

[35] Fu Q, Xie H, Zhou L et al.: Traditional Chinese medicine auricular point acupressure for the relief of pain, fatigue, and gastrointestinal adverse reactions after the injection of novel coronavirus-19 vaccines: a structured summary of a study protocol for a multicentre, three-arm, single-blind, prospective randomized controlled trial. Trials 2021;22: 162 .

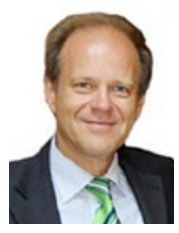

Univ.-Prof. Prof. mult. DI DDr. Gerhard Litscher

Leiter der Forschungseinheit für Biomedizinische Technik in Anästhesie und Intensivmedizin, der Forschungseinheit für Komplementäre und Integrative Lasermedizin

Chairman des TCM Forschungszentrums Graz, Medizinische Universität Graz

Auenbruggerplatz 39,EG19, 8036 Graz, Österreich,E-Mail:gerhard.litscher@medunigraz.at 